\title{
High Dielectric Constant in the Charge-ordered Manganese Oxide $\mathrm{CaMn}_{7} \mathrm{O}_{12}$
}

\author{
S. Yáñez Vilar ${ }^{a}$, A. Castro-Couceiro ${ }^{a}$, B. Rivas-Murias ${ }^{b}$, A. Fondado ${ }^{b}$, J. Mira ${ }^{b}$, J. Rivas ${ }^{b}$, M. A. Señarís- \\ Rodríguez $^{\mathrm{a}, *}$
}

a A Coruña/Spain, Química Fundamental, Universidad de A Coruña

b Santiago de Compostela/Spain, Física Aplicada, Universidad de Santiago

Received December 2nd, 2004; accepted February 15th, 2005.

\begin{abstract}
The dielectric properties of mixed-oxide $\mathrm{CaMn}_{7} \mathrm{O}_{12}$ are reported. This compound exhibits a high dielectric constant at room temperature $\left(\varepsilon_{\mathrm{r}}^{\prime}>10^{4}\right)$, for frequencies up to $10^{4} \mathrm{~Hz}$, values that make it very attractive for potential applications provided that its rather high losses can be minimized.

Complex plane analysis of the obtained data reveal an important extrinsic contribution to these high $\varepsilon^{\prime}{ }_{\mathrm{r}}$ values; and also that the
\end{abstract}

intrinsic dielectric constant of this material is $\varepsilon_{r, \infty}^{\prime} \sim 30$, a value rather high for this type of compounds, that could be related to the electronic process of charge-ordering present in this oxide below $440 \mathrm{~K}$.

Keywords: Dielectric properties; Mixed-oxides; Charge ordering

\section{Introduction}

Dielectric materials with high dielectric permittivities and low loss factors over a wide frequency range are always of great interest due to the important applications of such properties in many electronic devices, like high-performance capacitors. Nowadays, the best industrial performances are given by ferroelectrics, i.e., substances with permanent electrical dipoles, even in the absence of an external electric field [1].

Besides the "classical" and well-known ferroelectric perovskite materials $\left(\mathrm{BaTiO}_{3}, \mathrm{PbTiO}_{3}\right.$, etc.), that are the most widely used [1], other $\mathrm{ACu}_{3} \mathrm{M}_{4} \mathrm{O}_{12}$ (A: $\mathrm{Ca}$, etc.; $\mathrm{M}$ : Ti, $\mathrm{Fe}$, etc.) double-perovskites have been recently found [2]. Very interestingly, these latter display very high (giant) dielectric constants, $\left(\varepsilon_{\mathrm{r}} \sim 10^{4}\right.$ at $\left.300 \mathrm{~K}\right)$ whose intrinsic or extrinsic nature, as well as its origin is being currently intensively debated $[2,3]$.

In this work we focus in the dielectric properties of $\mathrm{CaMn}_{7} \mathrm{O}_{12}$, a closely related mixed-oxide that shows the added complexity of experiencing the electronic process of charge ordering as a function of temperature.

From the structural point of view the compound $\mathrm{CaMn}_{7} \mathrm{O}_{12}$ belongs to the $\left(\mathrm{AA}^{\prime}{ }_{3}\right) \mathrm{B}_{4} \mathrm{O}_{12}$ family, as it can be more readily seen when formally writing its formula as: $\left(\mathrm{CaMn}_{3}{ }^{3+}\right)\left(\mathrm{Mn}_{3}{ }^{3+} \mathrm{Mn}^{4+}\right) \mathrm{O}_{12}$. This perovskite-derived phase, consists of a threedimensional array of corner-sharing $\left[\mathrm{BO}_{6}\right]$ octahedra, that are tilted, so that one-fourth of the A-sites are distorted into icosahedra (that are occupied by

\footnotetext{
* Prof. Dr. Ma A. Señarís-Rodríguez Departamento Química Fundamental Facultad de Ciencias Universidad de A Coruña

E-15071 A Coruña/Spain

Tel: 34981167000

Fax: 34981167065

e-mail: tonasr@udc.es
}

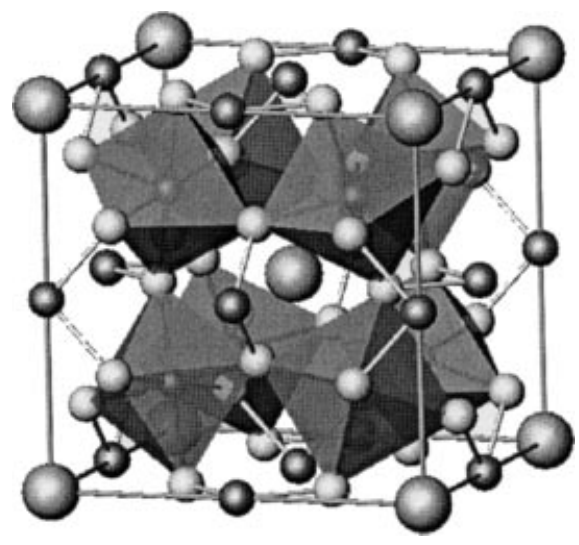

Figure 1 Cubic structure of $\mathrm{CaMn}_{7} \mathrm{O}_{12}(\mathrm{~T}>440 \mathrm{~K})$.

$\mathrm{Ca}^{2+}$ ions) and three-fourths into tetracapped rhombic prisms that accommodate the Jahn-Teller $\mathrm{Mn}^{3+}$ ions.

Above $440 \mathrm{~K}$, the formally $\mathrm{Mn}^{3+}$ and $\mathrm{Mn}^{4+}$ ions occupy at random the octahedral B-sites and the crystal symmetry is cubic $(\operatorname{Im} \overline{3})$ [4] (Figure 1). Nevertheless, at $440 \mathrm{~K}$ this compound experiences a structural phase transition associated to the electronic processes of charge and orbital ordering (CO) and (OO) [4]. Below $409 \mathrm{~K}$, the crystal structure has trigonal symmetry (Space group: $\mathrm{R} \overline{3}$ ) [5], due to the ordering of the distinguishable $\mathrm{Mn}^{3+}$ and $\mathrm{Mn}^{4+}$ ions in the B sublattice, (9d) and (3b) sites [5]. These two phases coexist over the temperature interval $409<\mathrm{T}<440 \mathrm{~K}$.

From the magnetic point of view, this compound is paramagnetic above $86 \mathrm{~K}$. Below $86 \mathrm{~K}$ long-range magnetic ordering of the magnetic moments of $\mathrm{Mn}^{3+}$ and $\mathrm{Mn}^{4+}$ ions has been observed, and between 86 and $50 \mathrm{~K}$ two different ordered magnetic phases have been detected: one ferrimagnetic and another modulated [6].

In this paper we study the dielectric behavior of this charge-ordered mixed oxide. 


\section{Experimental}

$\mathrm{CaMn}_{7} \mathrm{O}_{12}$ was synthesized by the so-called "liquid-mix" or Pechini method [7], using $\mathrm{CaCO}_{3}$ (Panreac, $>98,6 \%$ ) and $\mathrm{Mn}\left(\mathrm{NO}_{3}\right)_{2} \cdot \mathrm{H}_{2} \mathrm{O}$ (Aldrich, $>98 \%$ ) as starting materials. The procedure was as follows: We dissolved stoichiometric amounts of these metallic salts in a $1 \mathrm{M}$ citric acid aqueous solution. We then added the same volume of ethyleneglycol, and we finally diluted the so-obtained solution in water $(50 \% \mathrm{v} / \mathrm{v})$. The resulting solution was heated at $200{ }^{\circ} \mathrm{C}$ until a brown resin formed, whose organic matter subsequently decomposed at $400{ }^{\circ} \mathrm{C}$. The precursor powder was treated at $800^{\circ} \mathrm{C} / 48 \mathrm{~h}, 925^{\circ} \mathrm{C} / 48 \mathrm{~h}$ and $950^{\circ} \mathrm{C} / 48 \mathrm{~h}$, with intermediate grindings. Finally it was annealed at the highest temperature of $975^{\circ} \mathrm{C}$ (as for $\mathrm{T}>975^{\circ} \mathrm{C}$ the as-obtained sample melts) during $48 \mathrm{~h}$.

The structural characterization of the sample was carried out at room temperature by means of X-ray powder diffraction (XRPD) using a Siemens D-5000 diffractometer and $\mathrm{Cu}(\mathrm{K} \alpha)=1.5418 \AA$ radiation. The XRD data were analyzed by the Rietveld profile analysis using the Rietica software [8].

The morphology and particle size of this polycrystalline material was studied in a scanning electron microscope (SEM), JEOL 6400.

The complex dielectric permittivity of this material was measured with a parallel-plate capacitor coupled to a precision LCR meter Agilent $4284 \mathrm{~A}$, capable to measure in frequencies ranging from 20 to $10^{6} \mathrm{~Hz}$. The capacitor was mounted in an aluminium box refrigerated with liquid nitrogen, incorporating a mechanism to control the temperature up to $350 \mathrm{~K}$. The pelletized sample with average diameter of $2 \mathrm{~cm}$ was prepared to fit in the capacitor, and gold was sputtered on its surface to ensure good electrical contact with the plates of the capacitor. And aware of the controversy regarding extrinsic polarization effects as origin of very high dielectric constants in other ceramic materials $[9,10]$, additional measurements were performed using sputtered silver contacts.

Also, to test the optimal performance of the experimental set-up, a commercial $\mathrm{SrTiO}_{3}$ sample was measured and values similar to those reported in the literature [11] were obtained.

Complex plane analysis of the obtained impedance data was performed using the computer program LEVM [12].

\section{Results}

\subsection{Characterization}

The X-ray diffraction pattern shows that $\mathrm{CaMn}_{7} \mathrm{O}_{12}$ is the major phase in the sample but also that small amounts of $\mathrm{Mn}_{2} \mathrm{O}_{3}$ and $\mathrm{Mn}_{3} \mathrm{O}_{4}$ are present as secondary phases. Nevertheless, quantitative analysis of the XRD data by the Rietveld method indicate that these impurities represent minor amounts $(<2 \%)$.

This difficulty to obtain a completely pure phase has also been observed and highlighted by other authors, that prepared this mixed oxide by other methods and could neither obtain a single phase material [13].

As for the cell parameters of the $\mathrm{CaMn}_{7} \mathrm{O}_{12}$ phase, the refinements give $\mathrm{a}_{\mathrm{h}}=10.458 \AA$ and $\mathrm{c}_{\mathrm{h}}=6.342 \AA$ in good agreement with the data reported in the literature [13].

Morphologically this polycrystalline sample consists of an homogeneous distribution of pseudo-spherical particles of diameter $\approx 1 \mu \mathrm{m}$ as seen in the SEM micrographs.

\subsection{Dielectric properties}

The relative complex dielectric permittivity of the sample: $\varepsilon_{\mathrm{r}}=\varepsilon_{\mathrm{r}}^{\prime}-\mathrm{i} \varepsilon^{\prime \prime}{ }_{\mathrm{r}}\left(\varepsilon_{\mathrm{r}}=\varepsilon / \varepsilon_{0}\right.$; where $\varepsilon_{0}=8.85 \times 10^{-12} \mathrm{~F} / \mathrm{m}$ is the permittivity of free space) as well as its conductivity were measured as a function of frequency and temperature.

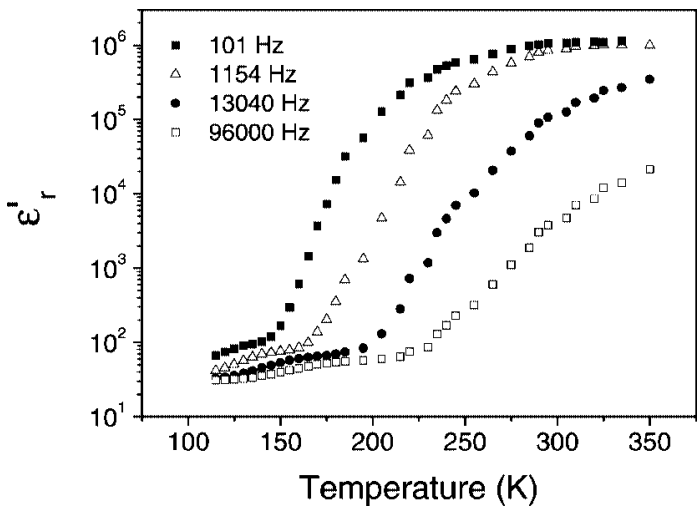

Figure 2 Temperature dependence of the real part of the dielectric permittivity $\left(\varepsilon_{\mathrm{r}}^{\prime}\right)$ measured at four different frequencies $(101 \leq$ $v(\mathrm{~Hz}) \leq 96000)$.

Figure 2 shows the real part of the dielectric permittivity $\varepsilon^{\prime}{ }_{r}$ (dielectric constant) as a function of temperature, obtained at different frequencies.

The first remarkable feature is that the value of $\varepsilon_{\mathrm{r}}^{\prime}$, that is of the order of 30-50 at low temperatures, experiences a significant increase above a certain temperature giving rise to a very high (giant) dielectric constant at room temperature $\left(\varepsilon_{\mathrm{r}}^{\prime}>10^{4}\right)$. The temperature at which this increase takes place markedly shifts to higher values as the measuring frequency increases.

The behavior of the real part of the dielectric permittivity as a function of frequency is shown in Figure 3. As it can be seen, the giant dielectric constant keeps a constant value for a certain frequency range giving rise to a "plateau", to then decrease as frequency gets higher. It is also interesting that this plateau extends over larger frequency ranges as temperature gets higher, so that for $\mathrm{T}=300 \mathrm{~K}$ this very high $\varepsilon_{\mathrm{r}}^{\prime}$ values $\left(\varepsilon_{\mathrm{r}}^{\prime} \sim 10^{6}\right)$ are retained up to $10^{4} \mathrm{~Hz}$.

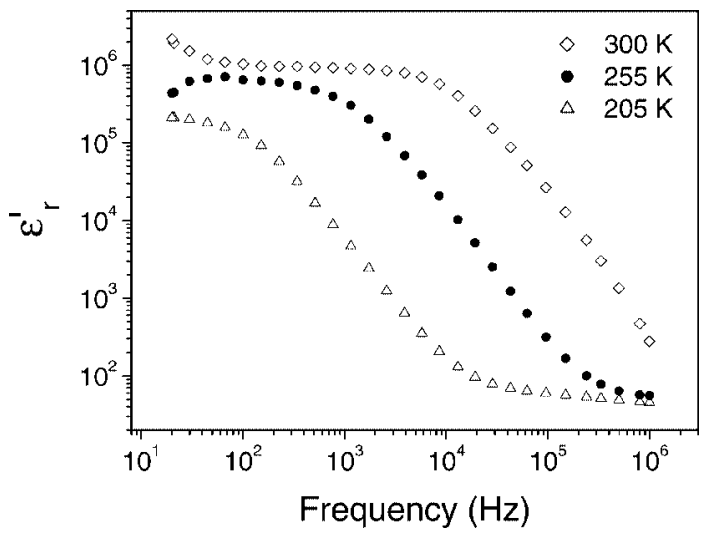

Figure 3 Frequency dependence of the dielectric constant measured at three different temperatures $(205 \leq \mathrm{T} \leq 300 \mathrm{~K})$. 


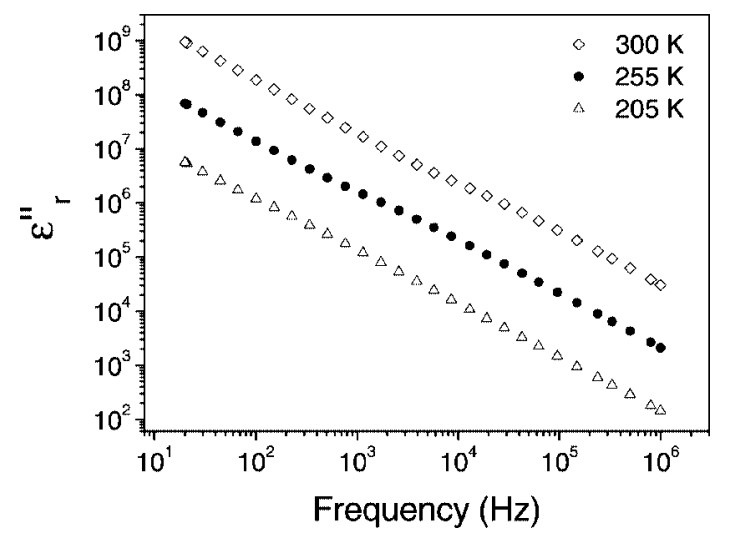

Figure 4 Frequency dependence of the imaginary part of the dielectric constant measured at three different temperatures $(205 \leq$ $\mathrm{T} \leq 300 \mathrm{~K})$.

Meanwhile, the imaginary part of the dielectric permittivity $\varepsilon^{\prime \prime}{ }_{r}$ shows rather high values, that decrease lineally with frequency and increase as temperature rises as shown in Figure 4. As a result, the values of the loss tangent $(\tan \delta)$ of this sample, $\tan \delta=\varepsilon_{\mathrm{r}}^{\prime \prime} / \varepsilon_{\mathrm{r}}^{\prime}$, are rather high (Figure 5).

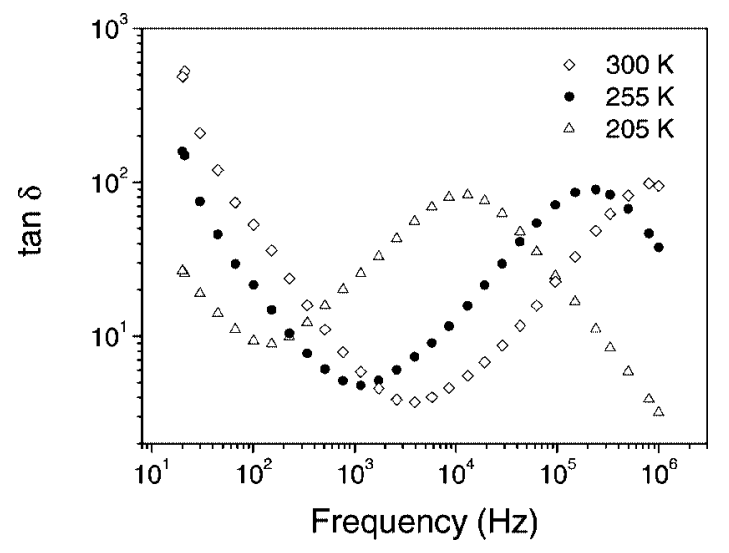

Figure 5 Frequency dependence of loss tangent measured at three different temperatures $(205 \leq \mathrm{T} \leq 300 \mathrm{~K})$.

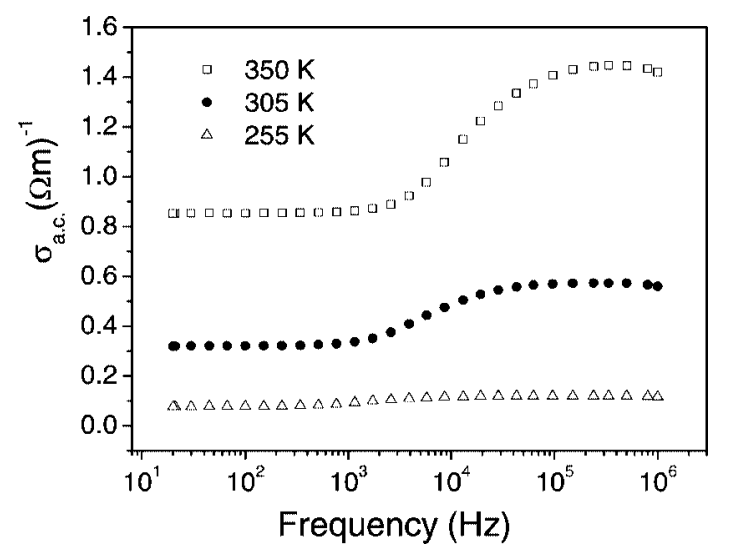

Figure 6 a. c. conductivity versus frequency in the temperature range $255 \leq \mathrm{T} \leq 350 \mathrm{~K}$.
On the other hand, and very importantly, this $\mathrm{CaMn}_{7} \mathrm{O}_{12}$ sample is not completely insulating but shows a certain conductivity that increases with temperature (Figure 6). In addition, for $\mathrm{T} \geq 300 \mathrm{~K}$ the low-frequency $\sigma_{\text {a.c. }}$ constant value experiences a sudden increase at $v \approx 10^{4} \mathrm{~Hz}$, that is in fact the frequency at which the dielectric constant shows the step-like decrease.

The here reported results correspond to data obtained using sputtered gold contacts.

If sputtered silver contacts are used slightly smaller $\varepsilon^{\prime}{ }_{\mathrm{r}}$ values are measured together with higher loss tangent values.

\section{Discussion}

The here reported dielectric behavior of the mixed oxide $\mathrm{CaMn}_{7} \mathrm{O}_{12}$ is very interesting both from the scientific and the technological point of view. In this context, the high dielectric constant of this compound at room temperature for frequencies up to $10^{4} \mathrm{~Hz}$ makes this material attractive for potential applications provided that its high dielectric losses can be minimized while keeping $\varepsilon^{\prime}{ }_{\mathrm{r}}$ as high as possible.

On the other hand, from the scientific point of view many questions should be understood about the origin of the observed dielectric behavior.

For this purpose, and taking into account that this material is not completely insulating, we have substracted from the $\varepsilon^{\prime \prime}{ }_{\text {r }}$ raw data the contribution from free charge carriers following equation [1]:

$\varepsilon_{\text {r,die }}^{\prime \prime}(\omega)=\varepsilon_{r}^{\prime \prime}(\omega)-\frac{\sigma_{\text {d.c. }}}{\varepsilon_{0} \omega}$

where $\varepsilon^{\prime \prime}{ }_{\text {rdie }}$ is the contribution of the dielectric to $\varepsilon^{\prime \prime}{ }_{\mathrm{r}}$ and $\sigma_{\text {d.c. }}$ is the d.c. electrical conductivity, that is obtained from the extrapolation of the conductivity, $\sigma(\omega)$, to low frequencies.

If we represent the so-obtained $\varepsilon_{\text {r,die }}^{\prime \prime}$ data as a function of frequency we find the results shown in Figure 7. As it

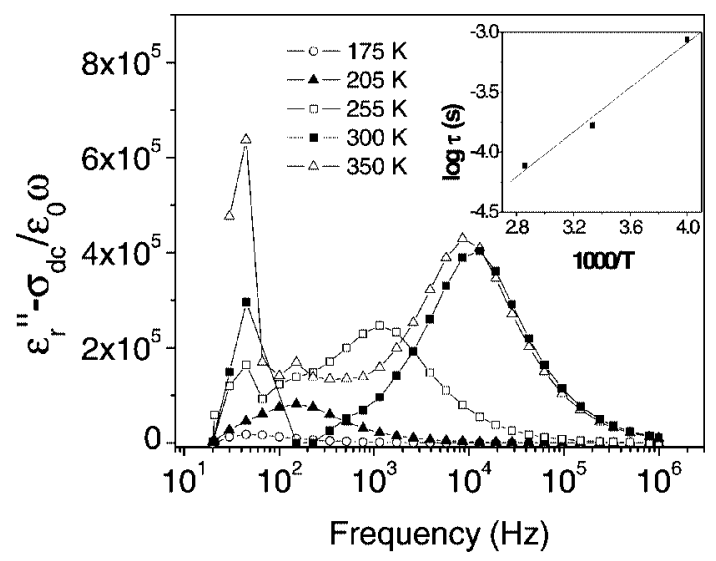

Figure 7 Frequency dependence of the imaginary part of the complex relative dielectric permittivity after substraction of the contribution from free charge carriers (Temperature range: $175 \leq \mathrm{T} \leq$ $350 \mathrm{~K}$ ). Inset: Logarithm of the characteristic time (inverse of the characteristic frequencies) versus the inverse of temperature. 
can be seen, in addition to the maximum found at very low frequency - that is typical of diffusional processes - for $\mathrm{T}>250 \mathrm{~K}$ a second maximum appears at $\sim 10^{4} \mathrm{~Hz}$, frequency at which the very high dielectric constant decreases in a step-like manner (Fig. 3). This second maximum ressembles that of a Debye-like dipolar relaxation process with characteristic times, $\tau=1 / \omega$, where $\omega$ is the characteristic frequency of relaxation.

A logarithmic fit of the characteristic relaxation times versus the inverse of temperature shows that these follow an Arrhenius type of behavior, $\tau=\tau_{0} \exp \left(\mathrm{U} / \mathrm{k}_{\mathrm{B}} \mathrm{T}\right)$, where $\mathrm{U}$ is the activation energy and $\mathrm{k}_{\mathrm{B}}$ the Boltzmann constant. From this fit we have obtained a $\mathrm{U} \sim 185 \mathrm{meV}$ and a $\tau_{0} \sim 153 \mathrm{~ns}$.

On the other hand, if we examine the temperature dependence of $\sigma_{\text {d.c. }}$ we observe a thermally activated behavior, with an activation energy of $216 \mathrm{meV}$.

This result correlates well with that of characteristics times establishing a link between its dielectric relaxation and its conductivity.

But these relaxation process, do they merely come from extrinsic effects due to interfacial polarization (from the electrode/sample interface, from grain boundaries in the polycrystalline sample) as described for other oxides in the literature $[14,15]$ or do they also have an intrinsic origin in the bulk material?

In this context, the fact that the results are dependent on the contact material already indicate a certain extrinsic contribution [16].

To try to separate extrinsic and intrinsic contributions we have carried out complex plane analysis of the obtained impedance data, which involve plotting the imaginary part $\left(Z^{\prime \prime}\right)$ against the real part $\left(Z^{\prime}\right)$ (Figure 8). The data have been best modelled on the basis of an equivalent circuit consisting of two parallel RC elements connected in series, that would represent the contribution from extrinsic factors (grain boundary and/or electrode response), while the bulk response has been approximated by another leaky capacitor, with a bulk capacitance determined by the intrinsic dielectric constant $\varepsilon_{\mathrm{r}, 1}^{\prime} \approx \varepsilon_{\mathrm{r}, \infty}^{\prime}$ and a bulk resistance that is frequency dependent.

This $\varepsilon^{\prime}{ }_{r, \infty}$ is of the order of 30 , a value that is still rather high for this type of oxides [17]. In this context it is interesting to note that in our research group we have also detected high dielectric constants in other oxides with charge ordering near their charge ordering temperature, such as in $\mathrm{Pr}_{0.67} \mathrm{Ca}_{0.33} \mathrm{MnO}_{3}[18]\left(\mathrm{T}_{\mathrm{CO}} \approx 225 \mathrm{~K}\right)$ were a four fold increase of its dielectric constant is observed as temperature decreases below $225 \mathrm{~K}$. All these results lead us to associate this enhanced intrinsic dielectric constants to the fact that the charge separation that occurs in a $\mathrm{CO}$ transition could lead to the formation of some kind of electrical dipoles in these type of materials, as it has also been theoretically proposed by other authors very recently [19].

More work is being done on samples of different particle size, with different geometries and thickness to better clarify the role of extrinsic contributions to the observed dielectric
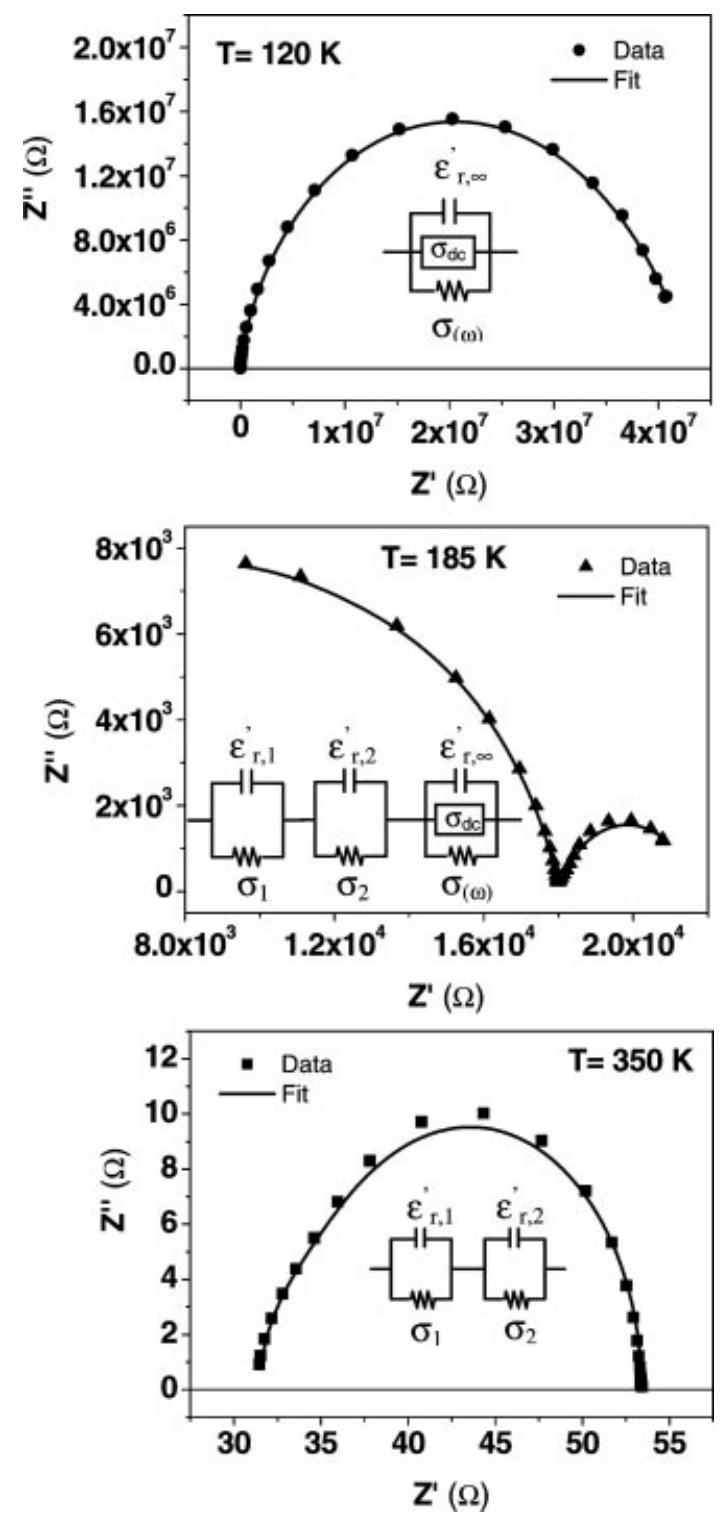

Figure 8 Impedance complex plane plot in three different temperature ranges: for $110<\mathrm{T}<150 \mathrm{~K}$ the $\mathrm{Z}^{*}$ plot contains a single arc with cero intercept at high frequencies. For $150<\mathrm{T}<250 \mathrm{~K}$ two arcs appear in the $\mathrm{Z}^{*}$ plot, while for $250<\mathrm{T}<350 \mathrm{~K}$ a single arc is again seen, but with a non zero intercept in this case. The experimental data have been fitted on the basis of an equivalent circuit consisting of three parallel $\mathrm{RC}$ elements connected in series.

response and to optimize the dielectric properties of this interesting material.

Acknowledgments. We thank financial support of the DGI of the Ministry of Science and Technology of Spain under project FEDER MAT 2001-3749.

\section{References}

[1] G. H. Haertling, J. Am. Ceram. Soc. 1999, 82, 797.

[2] M. A. Subramanian, L. Dong, N. Duan, B. A. Reisner, A. W. Sleight, J. Solid State Chem. 2000, 151, 323

[3] P. Lunkenheimer, R. Fichtl, S. G. Ebbinhaus, A. Loidl, Phys. Rev. B 2004, 70, 172102. 
[4] I. O. Troyanchuk, A. N. Chobot, Crystallogr. Rep. 1997, 42, 983.

[5] B. Bochu, J. L. Buevoz, J. Chenavas, A. Collomb, J. C. Joubert, M. Marezio, Solid State Commun. 1980, 36, 133.

[6] R. Przenioslo, I. Sosnowska, D. Hohlwein, T. Hauß, I. O. Troyanchuk, Solid State Commun. 1999, 111, 687.

[7] F. Licci, T. Besagni, L. Rinaldi, 1985 European Patent Appl. No. 85860253-2.

[8] C. J. Howard, H. Hunter, B. A. Rietica, A Computer Program for Rietveld Analysis of Organization Lucas Heights Research Laboratories.

[9] P. Lunkenheimer, T. Rudolf, A. Hemberger, S. Pimenov, S. Tachos, F. Lichtenberg, A. Loidl, Phys. Rev. 2003, 68, 245108.

[10] H. Lixin, J. B. Neaton, M. H. Cohen, D. Vanderbilt, C. C. Homes, Phys. Rev. B, 2002, 65, 214112.

[11] R. Viana, P. Lunkenheiner, J. Hemberger, R. Böhmer, A. Loidl, Phys. Rev. B 1994, 50, 601.
[12] J. Ross Macdonald, LEVM version 8.0 Comlex Nonlinear Squares Fitting Program, 2003.

[13] R. Przenioslo, I. Sosnowska, E. Suard, A. Hewat, A. N. Fitch, J. Phys. Condens. Matter 2002, 14, 5747.

[14] C. J. Maxwell, Electricity and Magnetism, Ed. Clarendon Press, Oxford, 1982.

[15] A. Von Hippel, Dielectric and Waves, Ed. Artech House, Boston, London, 1995.

[16] D. C. Sinclair, T. B. Adams, F. D. Morrison, A. R. West, Appl. Phys. Lett. 2002, 80(12), 2153.

[17] P. Lunkenheimer, V. Bobnar, A. V. Pronin, A. I. Ritus, A. A. Volkov, A. Loidl, Phys. Rev. B 2002, 66, 05215.

[18] F. Rivadulla, M. A. López Quintela, L. E. Hueso, C. Jardón, A. Fondado, J. Rivas, M. T. Causa, R. D. Sánchez, Solid State Commun. 1999, 110, 179.

[19] D. V. Efremov, J. Brink, D. I. Khomskii, Nature Mater. 2004, 3, 853 . 